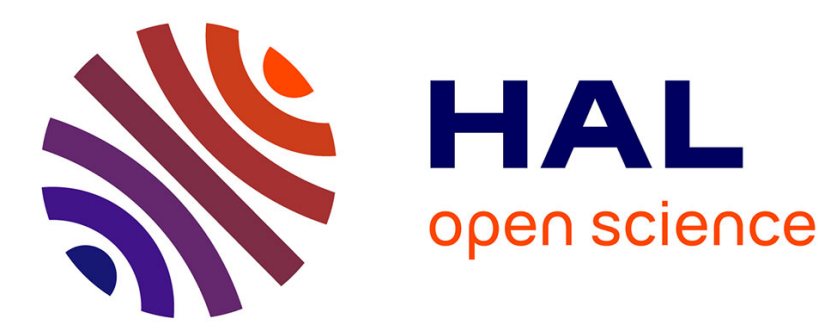

\title{
CAPITALISME ET MARCHÉ À LA RENAISSANCE Serge Walery
}

\section{To cite this version:}

Serge Walery. CAPITALISME ET MARCHÉ À LA RENAISSANCE: L'assimilation du capitalisme et du marché ou la négation de l'histoire. L'Économie politique, 2006, 2006/2 (30), pp.87-112. 10.3917/leco.030.0087 . hal-01224280

\section{HAL Id: hal-01224280 \\ https://hal.science/hal-01224280}

Submitted on 4 Nov 2015

HAL is a multi-disciplinary open access archive for the deposit and dissemination of scientific research documents, whether they are published or not. The documents may come from teaching and research institutions in France or abroad, or from public or private research centers.
L'archive ouverte pluridisciplinaire HAL, est destinée au dépôt et à la diffusion de documents scientifiques de niveau recherche, publiés ou non, émanant des établissements d'enseignement et de recherche français ou étrangers, des laboratoires publics ou privés. 


\title{
CAPITALISME ET MARCHÉ À LA RENAISSANCE
}

\author{
L'assimilation du capitalisme et du marché ou la négation de l'histoire
}

Même si le mot n'est apparu qu'à la charnière des 19 ème et 20ème siècles, au sein de l'école historique allemande, on sait, au moins depuis Ricardo et Marx, que le capitalisme est indissolublement lié à la perspective de l'accumulation. Au delà des aspects spécifiques mis en avant par chaque auteur, et des multiples apports qui ont complété et enrichi les bases posées au 19ème siècle, on peut considérer que la plupart des économistes s'accordent sur l'idée très vague et très générale selon laquelle le capitalisme est l'ensemble des éléments (comportements, activités, relations et/ou rapports sociaux, règles, institutions, conventions...) qui s'inscrivent dans la perspective de l'accumulation.

Le marché, lui, est, au niveau le plus élémentaire, le lieu de l'échange. Pour les économistes, il est l'"espace" (théorique, historique, géographique, institutionnel, conventionnel...) de confrontation de l'offre et de la demande ; dans une perspective théorique, il est également appréhendé comme un mode de coordination des choix individuels.

Sans doute n'est-il pas nécessaire d'aller au delà de cette esquisse très superficielle, ni d'avoir fait de longues études d'économie, pour noter que ces deux mots, potentiellement ces deux concepts, procèdent de deux "angles d'attaque", de deux perspectives, de deux points de vue distincts sur la réalité économique.

Pourtant, l'assimilation du capitalisme et du marché (ou de l'économie de marché) est sans doute l'une des confusions les plus courantes, non seulement dans les discours communs, mais aussi dans les discours savants. Elle constitue, par exemple, un des rares points de convergence des deux pôles violemment opposés du débat public sur la mondialisation, les "ultra-libéraux" et les "altermondialistes". II est cependant plus surprenant de retrouver une telle confusion dans des travaux d'économie ou d'autres sciences sociales. Ainsi, dans un ouvrage récent ${ }^{1}$ évoquant les relations entre la démocratie et le marché, Jean-Paul Fitoussi assimile-t-il systématiquement celui-ci au capitalisme ; il invoque par exemple, pour appuyer son raisonnement, des citations de Marx et de Schumpeter qui portent, elles, sur le capitalisme. ${ }^{2}$ On peut noter également que si cette assimilation est consciente, elle apparaît en quelque sorte "passive", l'auteur se contentant de prendre acte de sa banalité. Certains, au contraire, revendiquent clairement l'assimilation du capitalisme et du marché. C'est le cas d'Alain Caillé, qui réfute toute différence autre que d'échelle ou de degré entre marché et capitalisme ; le marché apparaissant, en quelque sorte, comme un " petit capitalisme ». ${ }^{3}$

Dans un souci de clarification, on se propose ici de mettre en évidence et de caractériser les relations entre capitalisme et marché durant la Renaissance, c'est à dire, au moment de l'émergence du capitalisme. II ne s'agit évidemment pas de prétendre que les relations ainsi mises à jour seraient immuables et caractériseraient encore l'économie contemporaine, mais de montrer que ces relations s'établissent entre deux ensembles non disjoints ${ }^{4}$ mais clairement distincts, et qu'en conséquence,

\footnotetext{
${ }^{1}$ FITOUSSI, J-P. La démocratie et le marché. Paris. Grasset, Nouveau Collège de Philosophie. 2004.

2 Ibid, pp 18-20.

3 Voir: CAILLÉ, A. Dé-penser l'économique ; contre le fatalisme. Paris. La Découverte (MAUSS). 2005. $\mathrm{p} 108$.

${ }^{4}$ Deux ensembles sont disjoints lorsqu'ils n'ont pas d'intersection, pas d'élément(s) commun(s).
} 
l'assimilation du capitalisme et du marché interdit de penser l'émergence du capitalisme commercial de la Renaissance.

Dès lors que l'on s'attache à une démarche théorique n'ignorant pas le concret, le terme de marché revêt, pour les économistes, une dualité qu'il convient d'éclairer quelque peu dès maintenant, dans la mesure où sa prise en compte est au cœur de notre démarche. Dans le cadre d'une réflexion sur les relations entre le capitalisme et le marché à la Renaissance (ou à toute autre époque), le marché peut être appréhendé d'un point de vue historique ou d'un point de vue purement théorique. Dans la mesure où personne ne saurait prétendre qu'existe, ou qu'a existé un jour, une société au sein de laquelle les marchés "fonctionnaient" comme le prévoit la théorie de l'Équilibre Général, ces deux points de vue, certes non disjoints, sont nécessairement distincts.

Si le marché est appréhendé d'un point de vue historique, il s'agit alors de s'interroger sur les relations entre le capitalisme émergent et les conditions (techniques, institutionnelles, conventionnelles...) historiques concrètes d'organisation des échanges à la Renaissance. Dans cette perspective, on parlera de lois historiques du marché, le terme de "loi" revêtant ici le sens de "propriété" ou de "caractéristique".

Si le marché est appréhendé d'un point de vue purement théorique, il s'agit alors de s'interroger sur les relations entre le capitalisme commercial de la Renaissance et les conditions nécessaires (la concurrence parfaite) à un fonctionnement efficace du marché, seul susceptible de permettre l'allocation optimale des ressources. Dans cette perspective, on parlera de lois théoriques du marché, le terme de "loi" revêtant ici le sens de "principe" ou de "précepte".

Dans un premier temps, on s'efforcera de montrer comment l'émergence du capitalisme commercial, au cours de la Renaissance, s'est faite en marge des lois historiques du marché. Plus précisément, on montrera comment le groupe social qui a "porté" l'émergence du capitalisme, les hommes d'affaires, s'est systématiquement efforcé de contourner et d'exploiter les lois historiques du marché de la Renaissance.

Dans un second temps, l'étude des stratégies concurrentielles des hommes d'affaires, et celle de l'organisation de la part des échanges qu'ils contrôlaient, permettront de montrer comment, aux origines du capitalisme, les impératifs de l'accumulation conduisirent les hommes d'affaires à adopter des comportements totalement antinomiques avec les lois théoriques du marché.

Il apparaîtra alors que le capitalisme des origines s'est constitué et développé "en dehors" des lois historiques et théoriques du marché, et qu'il entretenait avec celui-ci une relation de type parasitaire. De ce fait, appliquée à la Renaissance, l'assimilation du capitalisme et du marché se révèle être une absurdité aussi bien logique qu'historique.

Pour conclure, on s'interrogera brièvement sur les racines et les effets de l'assimilation contemporaine du capitalisme et du marché, ce qui conduira à dénoncer la mystification consciente ou inconsciente qu'elle constitue. 


\section{Capitalisme commercial et lois historiques du Marché}

\section{Cloisonnement des marchés et variabilité spatio-temporelle des prix}

Du point de vue des hommes d'affaires de la Renaissance, la première caractéristique ou propriété de l'organisation concrète des marchés à laquelle ils se trouvaient confrontés, était l'extrême cloisonnement spatial des marchés. Cette "loi historique" fut sans doute celle qui influa le plus directement sur leurs activités, et sur les modalités concrètes que revêtit alors l'accumulation du capital.

Le commerce au loin, et en particulier le commerce intercontinental, constituent une illustration presque caricaturale de ce que pouvaient être, à la Renaissance, le degré de cloisonnement des différents marchés, l'importance des écarts de prix qui en résultaient, et l'immensité des perspectives de profit que de tels écarts laissaient entrevoir. Cependant, le cloisonnement des marchés ne concernait pas seulement les échanges intercontinentaux, et apparaît de façon plus générale, comme une caractéristique globale essentielle de l'organisation matérielle des échanges marchands, à cette époque. En effet, quel qu'ait été le développement du grand commerce avant la Renaissance, les flux commerciaux de longue distance étaient demeurés trop étroits et trop irréguliers pour mettre en cause la très large prépondérance quantitative des échanges locaux et le cloisonnement des marchés, caractéristiques du tissu commercial hérité de l'Expansion Médiévale. D'ailleurs, même à la fin de la Renaissance, la faiblesse relative, la lenteur et l'irrégularité de la circulation économique à long rayon étaient encore telles, que pour la plupart des produits, les écarts de prix, dans le temps comme dans l'espace, demeuraient fréquemment de grande ampleur.

D'une part, à un instant donné, les prix d'un même produit en deux endroits distincts pouvaient être très différents. C'était bien sûr le cas des produits du commerce intercontinental, dont les prix en Europe étaient infiniment plus élevés que dans leurs pays d'origine. Mais cette variabilité spatiale des prix était aussi la règle à l'intérieur des "frontières" de l'économie-monde occidentale. Même après la Renaissance, et bien que, sous l'impulsion d'Amsterdam et des Provinces-Unies, le capitalisme commercial se soit considérablement développé, tant qualitativement que quantitativement, la variabilité spatiale des prix demeurait une donnée de base. Par exemple, en 1681, entre deux villes aussi impliquées dans les jeux du grand commerce que l'étaient Amsterdam et le grand port toscan de Livourne, le prix des cuirs russes variait du simple à plus du double. ${ }^{5}$ Plus généralement, le cloisonnement spatial des marchés à l'intérieur de l'économie-monde occidentale demeurait tel, qu'un même produit pouvait simultanément être surabondant dans une région et relativement rare dans une autre.

D'autre part, l'irrégularité importante de l'offre à l'échelle locale s'ajoutant au cloisonnement des marchés, les prix, en un lieu donné, de nombre de produits, connaissaient de très fortes variations dans le temps. Bien sûr, du fait de l'irrégularité de la circulation économique à longue distance, les marchandises ne faisant pas l'objet d'une production locale subissaient très directement cette variabilité temporelle des prix. Mais c'était aussi le cas d'un certain nombre de marchandises produites localement, et en particulier des produits de l'agriculture, les récoltes étant, par nature, très fluctuantes. ${ }^{6}$

\footnotetext{
5 Voir : BRAUDEL, F. Civilisation matérielle, économie et capitalisme. 3 volumes. Paris. Armand Colin. 1979. Tome 2, p 144.

6 Par exemple, lors de la grave pénurie céréalière qui affecta l'Italie en 1591, les prix des blés y étaient d'un tel niveau que des hommes d'affaires portugais d'Anvers réalisèrent des profits considérables (des concurrents, sans doute envieux, les estimèrent à 300\%) en s'approvisionnant en Mer Baltique. Pourtant, du fait des rapports prix/poids et prix/volume des produits concernés, et de l'importance relative des coûts de transport que cela impliquait, la rentabilité du commerce de céréales à long rayon était, en temps normal, plus qu'hypothétique.
}

Voir : BRAUDEL, F. Ibid. Tome 2, p 357. 
En dépit de l'essor de la circulation économique à long rayon, la forte variabilité spatiotemporelle des prix fut ainsi un trait majeur de l'univers commercial auxquels étaient confrontés les hommes d'affaires de la Renaissance.

\section{S'affranchir du cloisonnement pour exploiter la variabilité}

\section{Être informé}

Du point de vue des hommes d'affaires de la Renaissance, la variabilité spatio-temporelle des prix constituait une source presque inépuisable d'opportunités de profit. L'un des attraits majeurs du commerce au loin, dont ils firent leur domaine de prédilection, était justement que les écarts de prix y étaient à la fois considérables et relativement stables. Cependant, leur recherche inlassable du profit les conduisit naturellement à s'intéresser à tout écart de prix suffisamment important pour offrir des perspectives d'accumulation alléchantes, et tel qu'il leur soit matériellement possible de l'exploiter. Dans cette optique, la première condition matérielle que devaient satisfaire les hommes d'affaires, pour mener à bien leurs activités, était de connaître les écarts de prix et d'en prévoir les évolutions. II leur fallait s'affranchir du cloisonnement informationnel caractéristique des marchés de l'époque. La rentabilité des opérations qu'ils entreprenaient, dépendait en effet très directement de la précision et de la fiabilité des informations dont ils disposaient, sur les prix concernés, et surtout sur les multiples facteurs ou évènements susceptibles de les faire varier pendant la durée, souvent importante, de l'opération envisagée.

Pour un homme d'affaires ou une société d'envergure, la quantité et la diversité des paramètres qu'il était nécessaire de connaître pour garantir, autant que possible, la profitabilité de ses activités, étaient, eu égard aux normes de l'époque, incommensurables. II était tout d'abord indispensable de se tenir précisément informé des prix, des cours de change et des taux d'intérêt, sur chacun des marchés sur lesquels on avait coutume d'intervenir, mais aussi sur tous les autres marchés susceptibles d'offrir, ne serait-ce que temporairement, des opportunités intéressantes. En outre, du fait de la lenteur de la circulation économique et de la durée des opérations qu'elle impliquait, l'anticipation des variations de prix, c'est-à-dire celle des mouvements de l'offre et de la demande sur chaque marché, revêtait un caractère crucial ; il fallait donc être informé aussi complètement que possible des multiples mouvements de marchandises en cours ou en préparation, mais aussi de l'évolution des modes et des goûts locaux, dont l'influence sur la demande de nombreux biens était importante. II était aussi essentiel de disposer de renseignements complets et réguliers sur les évènements de tous ordres déterminant le degré de risque pesant sur chaque opération envisagée, et/ou susceptibles de provoquer des variations de prix soudaines. Les hommes d'affaires devaient donc s'efforcer de se tenir au courant des conflits armés et des actes de piraterie qui pouvaient affecter la sécurité de la circulation économique, ainsi que des évènements et troubles politiques, administratifs, diplomatiques, ou même sanitaires et climatiques, qui pouvaient entraîner des blocages, des retards et des difficultés de tous ordres.

Le volume et la diversité de l'information dont les hommes d'affaires de la Renaissance souhaitaient disposer pour mener à bien leurs activités, n'avaient ainsi pas plus de limites que les profits qu'ils désiraient réaliser. On pourrait dire, de façon un peu frappante et donc peu nuancée, que dans les conditions historiques de l'époque, l'accumulation du capital était contrainte ou bornée par celle de l'information. La logique d'accumulation imposait donc aux hommes d'affaires de la Renaissance une stratégie visant à transcender le cloisonnement spatial et informationnel des marchés de l'époque ; elle les poussait à s'affranchir d'une des lois historiques prépondérantes du Marché, afin d'exploiter les opportunités de profit que celle-ci engendrait.

Pour mettre en œuvre cette stratégie, les hommes d'affaires de la Renaissance consacrèrent une énergie et des investissements considérables à constituer un système d'information étonnamment performant, et répondant, autant que le permettaient les conditions matérielles et techniques de 
l'époque, aux impératifs de l'accumulation. Reprenant en cela le vocabulaire habituellement utilisé par les historiens de la période, on désignera par le terme de "courrier", ${ }^{7}$ cet ensemble de moyens très divers développés par les hommes d'affaires. ${ }^{8}$

\section{Des réseaux}

Très tôt, le courrier devint l'une des activités quotidiennes prépondérantes des hommes d'affaires. Pour avoir connaissance du plus grand nombre possible d'opportunités d'accumulation et être ainsi en mesure de sélectionner et de réaliser les plus intéressantes, les hommes d'affaires devaient pouvoir compter sur de nombreux informateurs, entretenir avec eux des relations de confiance, organiser la circulation des lettres que ceux-ci rédigeaient, lire quotidiennement ces multiples lettres, prendre note des informations fournies et les analyser, rédiger des instructions ou des demandes de renseignements particuliers, et, bien sûr, s'occuper de les faire parvenir à leurs destinataires. Concrètement, ces tâches, lourdes mais indispensables, répondaient à deux impératifs : trouver l'information et la faire circuler.

Afin de traquer l'information, les hommes d'affaires mirent peu à peu sur pied de vastes réseaux d'informateurs, dont l'activité tendait bien souvent à s'apparenter à du renseignement ou à ce qu'on appelle aujourd'hui la "veille" ou l'intelligence" économique. ${ }^{9}$ Les informateurs sur lesquels pouvaient s'appuyer les sociétés importantes étaient, en premier lieu, le personnel des filiales et succursales, pour lequel la recherche et la collecte d'informations de toutes sortes étaient une tâche prioritaire. De même, les commissionnaires avec lesquels on avait l'habitude de traiter, ou les hommes d'affaires originaires d'autres places avec lesquels on collaborait plus ou moins régulièrement, étaient de précieuses sources d'informations. Mais on s'efforçait également d'exploiter tous les liens ou relations qui, a priori, ne relevaient pas, ou pas seulement, du monde des affaires. Ainsi, sur les places "étrangères", la communauté d'origines pouvait se révéler féconde, même avec des "compatriotes" n'exerçant pas d'activités commerciales. On pouvait pareillement s'appuyer sur des liens familiaux, même indirects ; par exemple, entretenir des relations épistolaires régulières avec un parent éloigné exerçant des fonctions administratives dans un comptoir ou une possession extérieurs à l'économiemonde occidentale, pouvait constituer le support informationnel d'opérations de commerce au loin particulièrement rentables.

Encore fallait-il être en mesure de faire circuler les informations ainsi recueillies, ce qui, dans les conditions historiques et techniques de l'époque, revenait à transporter des lettres. Or, étant donné l'échelle spatiale des activités des hommes d'affaires et des réseaux qu'ils mirent sur pied, le relativement faible degré de développement des systèmes de transports faisait de l'organisation de la circulation des lettres d'affaires, une entreprise de grande envergure. Dans la mesure où il n'était pas

7 Dans la plupart des cas le mot courrier prendra cette acception lorsqu'il sera précédé d'un article défini singulier. Précédé d'un article indéfini ou pluriel, il désignera le plus souvent les "porteurs de dépêches". On privilégiera le terme de lettre pour évoquer ce que transportaient les courriers.

8 Pour une approche plus détaillée et plus concrète du rôle et de la place du courrier dans les activités et pratiques des hommes d'affaires, voir : WALERY, S. Communication et accumulation du capital ; pour une perspective de longue durée. In : Quaderni. CREDAP. Université Paris-IX-Dauphine. Hiver 1990/1991. pp 15-35.

9 Par exemple, au début du XVI ${ }^{\text {ème }}$ siècle, alors que la mise en exploitation de la route des Indes mettait un terme à la domination quasi-monopolistique de Venise sur le commerce des épices, on était, à Lisbonne, très précisément et régulièrement informé des quantités, des prix et de la qualité des différentes épices que les galères vénitiennes ramenaient d'Alexandrie ou de Beyrouth.

Voir : GODINHO, V. M. Les découvertes. $X V^{e ̀ m e}-\left.X V\right|^{e ̀ m e}$ : une révolution des mentalités. Paris. Éditions Autrement, Série Mémoires, supplément au n¹. 1990. 
toujours possible, ni surtout souhaitable, de faire circuler les lettres avec les marchandises, il était nécessaire de disposer de nombreux courriers, de les rémunérer et de payer leurs frais (alimentation, hébergement, moyens de transport...) sur des durées parfois importantes, de choisir, au gré des circonstances du moment et des destinations, les routes les plus pratiques et les plus sûres, de prévoir et d'organiser des haltes pour les courriers terrestres ainsi que des relais lorsqu'ils se déplaçaient à cheval, ce qui devint de plus en plus fréquent, d'affréter des embarcations légères pour les liaisons maritimes... Bref, pour un homme d'affaires ou une société d'envergure internationale, l'organisation matérielle de la circulation des informations entre les multiples points de son réseau était une lourde tâche, dont le coût était considérable. ${ }^{10}$

Le coût de la circulation de l'information, en particulier lorsqu'on la souhaitait rapide, explique qu'à l'exception des princes, seuls les hommes d'affaires les plus importants aient alors été en mesure de mettre sur pied des réseaux postaux de grande échelle. II explique aussi pourquoi les hommes d'affaires furent très tôt conduits à regrouper leurs forces en matière de circulation du courrier, pratique qui allait peu à peu déboucher sur la constitution de sociétés de services postaux. Tout d'abord, dans la mesure où il était extraordinairement difficile et coûteux d'organiser soi-même la circulation de tout son courrier et vers toutes les destinations, et du fait que cela permettait un partage des coûts, il devint très vite habituel de transporter le courrier des autres ; lorsqu'un homme d'affaires organisait pour son propre compte un "départ" vers une destination donnée, il faisait au préalable le tour des autres hommes d'affaires de la place, afin de se charger, moyennant rémunération ou service réciproque, des lettres que ceux-ci souhaitaient faire parvenir à la même destination. L'étape suivante fut franchie lorsque ces collaborations, souvent régulières, s'institutionnalisèrent, plusieurs hommes d'affaires ou sociétés d'une même place s'associant pour organiser et mettre sur pied un système commun de services postaux, susceptible de satisfaire l'essentiel des besoins de chacun des associés. Naturellement, ces associations se chargeaient volontiers, moyennant rémunération, de transporter d'autres lettres que celles des associés ; elles étaient ainsi de véritables sociétés de services postaux. Ce fut, par exemple, le cas de la Scarcella, société fondée dès 1357 à Florence par une quinzaine ${ }^{11}$ de maisons commerciales locales, et qui assurait même des services réguliers sur certaines destinations telles que Gênes, Avignon ou Venise...

\section{La quête de la vitesse}

Cependant, comme une grande part des activités et instruments développés par les hommes d'affaires de la Renaissance, le courrier vit son efficacité globale étroitement contrainte par la lenteur, l'irrégularité et les multiples faiblesses des transports de l'époque. Or, du point de vue de l'accumulation du capital, le temps de circulation de l'information était non seulement un temps mort, mais aussi un important facteur d'incertitude et de risque. Les hommes d'affaires ne pouvaient se contenter de fonder leurs décisions sur les informations toujours plus ou moins périmées dont ils disposaient ; il leur fallait, sur la base de ces informations, prévoir, ou pour mieux dire anticiper, les variations de prix. Et, plus longue était la durée de circulation de l'information, plus long était le terme des anticipations, plus grand était leur degré d'incertitude. Dans ces conditions, on conçoit que la vitesse de circulation de l'information et sa régularité aient été des enjeux importants pour les hommes d'affaires de la Renaissance, et qu'ils se soient sans cesse efforcés de les accroître.

10 «La nouvelle, marchandise de luxe, vaut plus que son pesant d'or.»

Voir : BRAUDEL, F. La Méditerranée et le monde méditerranéen à l'époque de Philippe II. 2 volumes. Paris. Armand Colin. 1966. (6 ${ }^{\text {ème }}$ édition, 1986). Tome 1, p 335.

11 Une douzaine selon Yves Renouard (in : RENOUARD, Y. Les hommes d'affaires italiens du Moyen Âge. Paris. Armand Colin. 1949. p 138), et dix-sept selon Jean Favier (in : FAVIER, J. De l'or et des épices ; naissance de l'homme d'affaires au Moyen Âge. Paris. Fayard. 1987. p 87). 
Même si le développement des services postaux et de leur efficacité ne fut pas le seul fait des hommes d'affaires, ceux-ci, par leurs efforts d'organisation, par leurs investissements et par les progrès qui en résultèrent, furent indiscutablement les premiers et les principaux acteurs de ce mouvement. C'est ainsi que les réseaux d'acheminement du courrier qu'ils mirent en place, étaient sensiblement plus efficaces et plus rapides que ceux créés pour le service des princes. ${ }^{12}$ De façon plus générale, et bien que l'extrême pauvreté des sources concernant le Moyen Âge ne permette pas d'en fixer l'ordre de grandeur, il ne fait aucun doute que l'organisation rationnelle des services postaux permit, à ceux qui avaient les moyens d'y recourir, un accroissement significatif de la vitesse de circulation de l'information, si ce n'est de sa régularité. ${ }^{13}$ Ainsi, les moyens matériels mis en œuvre par les hommes d'affaires de la Renaissance, et à un moindre degré par les princes, leur permettaient-ils de faire circuler les informations à une vitesse inconcevable pour la plus grande part des populations occidentales de l'époque. Pourtant, à l'échelle de l'horizon spatial des hommes d'affaires, et au regard de l'importance que revêtait la vitesse de circulation de l'information dans la perspective de l'accumulation, les performances réalisées apparaissent sensiblement moins brillantes. ${ }^{14}$

Sans doute est-ce l'absence d'innovations techniques radicales en matière de transport qui explique ce bilan quelque peu mitigé. Si les efforts d'organisation déployés par les hommes d'affaires débouchèrent sur une incontestable accélération de la circulation de l'information, celle-ci n'en demeura pas moins soumise à la pesanteur des techniques. D'ailleurs, une fois engrangés les résultats de l'amélioration organisationnelle des services postaux, c'est-à-dire à partir du XVlème siècle, la vitesse de circulation de l'information tendit à se stabiliser, et ne connut guère d'évolution significative jusqu'au XVIIIème siècle.

12 C'est ainsi qu'à partir du XIV ème siècle, la papauté recourut très régulièrement aux services des grandes maisons commerciales florentines, en particulier pour sa correspondance urgente, ou qu'à la charnière des $X V^{\text {ème }}$ et $X V l^{\text {ème }}$ siècles, I'Empereur du Saint-Empire, Maximilien $\left.\right|^{\text {er }}$, confiait fréquemment ses lettres au réseau postal de Jacob Fugger, en dépit du service spécialement mis en place par les Comtes de Taxi pour les besoins du gouvernement impérial.

Voir : SCHICK, L. Un grand homme d'affaires du XVI ${ }^{\text {ème }}$ siècle : Jacob Fugger. Paris. S.E.V.P.E.N. 1957. p 237.

A titre d'ordre de grandeur, au début du XVIème siècle, alors qu'il ne fallait jamais moins de quinze jours aux courriers de la couronne espagnole pour effectuer le trajet de Bruxelles à Madrid, ceux des hommes d'affaires le faisait généralement en onze jours ; en outre, alors qu'un courrier royal quittait Bruxelles toutes les trois semaines, les maisons commerciales organisaient, au minimum, un départ hebdomadaire.

Voir: VASQUEZ de PRADA, V. Lettres marchandes d'Anvers. 4 volumes. Paris. S.E.V.P.E.N. 19601961. Tome 1, p 41.

13 Sur la base de données portant sur la correspondance anversoise du début du XVlème siècle, on peut estimer que, sur les itinéraires fréquentés, les lettres confiées aux soins des hommes d'affaires anversois se déplaçaient, en moyenne, d'une quarantaine de kilomètres par jour lorsque les courriers étaient à pied, et de 60 à 80 kilomètres par jour lorsque ceux-ci étaient à cheval.

Voir : VASQUEZ de PRADA, V. Op cit. Tome 1, p 38.

À la même époque, la vitesse moyenne observée sur le trajet Venise-Rome était de l'ordre de 100 kilomètres par jour, mais tombait dans certains cas à moins de 50 kilomètres par jour, alors que le "record" s'établissait à 36 heures, soit plus de 250 kilomètres par jour.

D'après des données fournies par: SARDELLA, P. Nouvelles et spéculations à Venise au début du $\left.X V\right|^{e m e}$ siècle. Paris. Armand Colin. 1948. pp 56-57.

14 Comme le disait si poétiquement Fernand Braudel :

«L'homme peut attaquer l'espace à sa guise, faire sauter en éclats les rames des galères renforcées, ou crever les chevaux de relais, ou se donner l'illusion par bon vent de voler sur la mer, l'espace, en fait, lui résiste par son inertie et, au-delà de ces exploits fugitifs, reprend chaque jour ses revanches.»

Voir : BRAUDEL, F. La Méditerranée et le monde méditerranéen à l'époque de Philippe II. Op cit. Tome 1, p 339. 
Des moyens à la mesure de l'enjeu

D'un point de vue quantitatif, les efforts déployés par les hommes d'affaires se révélèrent beaucoup plus fructueux. Les réseaux d'informateurs et les services postaux qu'ils développèrent, furent très rapidement en mesure de faire circuler, à l'échelle de l'économie-monde occidentale, des quantités d'informations considérables, et ce malgré les contraintes et obstacles de tous ordres, déjà évoqués.

Bien sûr, à l'exception des nouvelles exceptionnelles, déterminantes et particulièrement urgentes, il était aisé, et ni les hommes d'affaires ni leurs informateurs ne s'en privèrent, de multiplier le nombre d'informations contenues dans chaque lettre. Bon nombre de lettres d'affaires étaient ainsi de véritables notes de conjoncture économique, recensant de nombreuses données quantitatives : prix de multiples produits, taux de change et d'intérêt, unités monétaires, droits de douane, poids et mesures, règlements administratifs... ${ }^{15}$

Cependant, c'est avant tout en se dotant peu à peu des moyens matériels de faire circuler d'énormes quantités de courrier, que les hommes d'affaires de la Renaissance assouvirent leur boulimie d'information. La quantité de lettres d'affaires qui circulaient dans l'économie-monde occidentale de l'époque était telle, qu'étant donné le nombre relativement limité des acteurs concernés, on a quelque peine à imaginer comment s'organisait concrètement l'émission (la rédaction), la circulation et la réception (la lecture et l'analyse) d'une telle masse de courrier, et on réalise beaucoup mieux à quel point les activités liées au courrier accaparaient les hommes d'affaires et leurs employés. Dès la fin du XIVème siècle dans les régions les plus dynamiques, tout homme d'affaires de quelque envergure recevait ou expédiait plusieurs dizaines de lettres, chaque jour ouvrable. A cet égard, l'exemple le plus fréquemment cité 16 est celui d'un homme d'affaires toscan de Prato, Francesco di Marco Datini, qui, au regard des grandes dynasties d'affaires florentines, n'était qu'un homme d'affaires de second rang. Au cours de ses dix années les plus actives, entre 1395 et 1405, il expédia ou reçut plus de 80000 lettres, qu'il archiva soigneusement dans sa vaste et luxueuse demeure.

Bien que les individus évoluant dans l'univers du grand commerce aient alors été en nombre relativement limité, et en dépit des si lourdes contraintes qu'imposait l'incommensurabilité de l'espace dans lequel elles s'efforçaient de circuler, ce sont, chaque année, plusieurs millions de lettres d'affaires qui s'échangeaient au sein de l'économie-monde occidentale ou entre celle-ci et les nouveaux mondes ; millions de lettres qui permirent aux hommes d'affaires de s'affranchir du cloisonnement informationnel des marchés, c'est à dire, d'agir, pour leur plus grand profit, en marge d'une des lois historiques fondamentales du marché de la Renaissance.

15 Certaines lettres d'affaires étaient de véritables études de marché, telle cette Information sur le poivre, les îles Moluques et les îles de Bamda, qui parvint à Lisbonne au début des années 1540, et dans laquelle l'auteur estime d'abord les quantités de clous de girofle, de macis et de noix de muscade, produites par les différentes îles de cet archipel où le poivre était relativement rare, et dresse la liste des différents prix ; puis, après avoir comparé ceux-ci avec les prix pratiqués sur la côte occidentale de l'Inde, où le poivre était abondant et les épices précitées plutôt recherchées, il étudie très précisément les coûts et les recettes prévisibles d'un éventuel trafic visant à tirer profit du cloisonnement de ces deux marchés.

Voir : GODINHO, V.M. Op cit. pp 40-41.

16 Voir, entre autres :

- RENOUARD, Y. Op cit. pp 160-167.

- FAVIER, J. Op cit. $\mathrm{p} 89$.

- TENENTI, A. Les affaires et l'argent. In : CLOULAS, I. (coordination de). L'Italie de la Renaissance. Paris. Fayard. 1990. p 303. 


\section{S'affranchir des lois historiques de la concurrence}

Il est une autre catégorie majeure de lois historiques du marché que les hommes d'affaires de la Renaissance contournèrent très rapidement, celles ayant trait aux formes de la concurrence. En effet, dans l'organisation des marchés héritée de l'Expansion Médiévale, l'exercice de la concurrence était étroitement encadré par une multitude de règlements administratifs ou corporatifs, qui représentaient autant de contraintes pesant sur les profits et l'accumulation.

Cette réglementation, dont l'application par les autorités administratives était parfois très tatillonne, et qui visait à garantir l'égalité des vendeurs, à protéger les intérêts des acheteurs et à éviter les fraudes de toutes sortes, débouchait concrètement sur une concurrence très organisée, et d'un degré relativement limité. Tout d'abord, afin de faciliter l'exercice d'un tel contrôle et de garantir une certaine équité ou loyauté de la concurrence, les autorités administratives s'efforçaient inlassablement d'assurer le caractère public des transactions commerciales. A cet effet, les lieux et horaires des foires et marchés étaient très précisément définis, et les transactions réalisées en dehors du cadre spatiotemporel ainsi fixé donnaient lieu à différentes sanctions (amendes, confiscations...), lorsqu'elles parvenaient à la connaissance des autorités. Dans le même esprit, mais aussi afin de permettre aux acheteurs de prendre le temps d'étudier les différentes propositions et de mûrir leurs décisions, les foires étaient le plus souvent divisées en plusieurs périodes :17 durant les "jours de montre", les vendeurs présentaient leurs produits et négociaient avec les acheteurs potentiels, mais aucune transaction ne pouvait être effectivement conclue avant les "jours d'issue". L'équité de la concurrence et la protection des consommateurs justifiaient également une réglementation et un contrôle très strict de la qualité des produits, et des poids et mesures utilisés lors des transactions. Enfin, en particulier sur certains marchés et dans certains États ou principautés, les prix eux-mêmes pouvaient faire l'objet d'une réglementation ; en effet, le souci du bien public conduisaient parfois les autorités à fixer, soit des prix minimaux pour sauvegarder les intérêts des producteurs en période de surabondance, soit, au contraire, des prix maximaux pour protéger les consommateurs des effets de la spéculation en période de pénurie.

Cependant, ces multiples contraintes qui limitaient très sensiblement la marge de manœuvre des acteurs de l'échange ne pesaient pas de façon uniforme sur tous les domaines de l'activité commerciale. D'une part, le souci qu'avait les autorités administratives de protéger l'ensemble des consommateurs et des membres des corporations les conduisit naturellement à s'intéresser en priorité aux produits d'usage courant et aux échanges locaux ; en revanche, elles se préoccupèrent sensiblement moins des produits de luxe auxquels seules les classes les plus aisées pouvaient accéder, et des produits "exotiques" qui n'entraient pas en concurrence directe avec les productions locales. D'autre part, les différents types de règlements précédemment évoqués, et en particulier le caractère obligatoirement public des échanges, s'appliquaient avant tout aux transactions commerciales au comptant, et, de fait, bien des transactions à crédit y échappaient largement ; par exemple, lorsqu'un marchand de vins achetait à terme la récolte d'un domaine viticole. Par ailleurs, l'attitude générale des autorités administratives quant à la réglementation et au contrôle des échanges, n'était, elle non plus, pas uniforme. L'influence et la participation de l'élite des affaires au sein des autorités administratives (cités, principautés ou États-Nations en gestation) étaient en effet très variables.

Afin de s'affranchir des lois historiques de la concurrence qui limitaient les perspectives et les potentialités d'accumulation, les hommes d'affaires furent donc naturellement conduits à privilégier les

17 Sur l'organisation concrète des foires commerciales, voir, par exemple :

- BOURQUELOT, F. Études sur les foires de Champagne, sur la nature, l'étendue et les règles qui s'y faisaient aux XIPेme $X I I \hat{P}^{m e}$ et XIV ème siècles. 2 volumes. Paris. Imprimerie Nationale. 1865-1866.

- BRÉSARD, M. Les foires de Lyon aux XVème et XV'户̀me siècles. Paris. A. Picard. 1914.

- VARILLE, M. Les foires de Lyon avant la Révolution. Lyon. L. Bonnaviat. 1920. 
places commerciales ${ }^{18}$ et à développer les domaines d'activités, les moins exposés à la réglementation administrative : le grand commerce et le commerce au loin dont l'organisation était sensiblement moins contrainte, dans la mesure où ces échanges, souvent difficilement contrôlables, ne portaient que sur des quantités relativement limitées et n'impliquaient pas la masse des consommateurs et des producteurs $^{19}$; le crédit et la finance, qui, du fait de la condamnation de l'Église relayée par un certain nombre d'interdictions administratives, conservaient à l'époque un caractère illicite ou pour mieux dire souterrain, ce qui excluait que les autorités administratives en définissent et en contrôlent l'organisation.

Avec le rôle joué par le commerce au loin et la finance dans l'accumulation, c'est ainsi une part prépondérante des activités des hommes d'affaires qui se développèrent en marge des lois historiques de la concurrence, auxquelles la plus grande part des échanges de l'époque continuait d'être soumise.

\section{Capitalisme commercial et lois théoriques du Marché}

Si les lois historiques du marché "en vigueur" à la Renaissance définissaient une organisation concrète des marchés bien peu conforme à l'idéal walrassien, les efforts déployés par les hommes d'affaires pour s'en affranchir ne visaient aucunement à promouvoir cet idéal. Bien au contraire, étant donné les modalités concrètes de l'accumulation à l'époque, le respect des lois théoriques de la concurrence aurait été tout aussi incompatible avec l'accumulation que ne l'aurait été celui des lois historiques.

On a vu que c'est essentiellement à travers l'exploitation commerciale des écarts de prix générés par le cloisonnement des marchés, que les hommes d'affaires de la Renaissance s'efforcèrent d'étancher leur soif de profit. Or, comme l'illustrent les procédés d'arbitrage, l'existence, entre les hommes d'affaires, de pratiques concurrentielles conformes aux lois théoriques du marché, aurait, par nature, engendré un mouvement de réduction des écarts de prix et de décloisonnement des marchés, c'est-à-dire, une régression de la profitabilité des opérations commerciales menées, un rétrécissement des opportunités et des perspectives d'accumulation. Autrement dit, dans les conditions historiques de la Renaissance, et du point de vue des hommes d'affaires, le rythme potentiel d'accumulation était inversement proportionnel au degré effectif de concurrence pesant sur les activités commerciales qu'ils menaient.

C'est pourquoi, loin de promouvoir une concurrence parfaite, les hommes d'affaires essayaient au contraire de faire en sorte que leurs activités échappent, autant que possible, à toute forme de concurrence. Matériellement, cette volonté d'agir en marge des lois théoriques du marché se traduisit

\footnotetext{
18 C'est ainsi qu'au début du XVI ${ }^{\text {ème }}$ siècle, lorsque les grandes découvertes et le basculement vers l'ouest du commerce au loin provoquèrent l'irrémédiable déclin de Venise, le rôle de plaque tournante du commerce au loin fut désormais joué par Anvers, et non par Bruges, cité pourtant beaucoup plus importante, et qui disposait déjà d'une longue tradition d'affaires. Certes, l'ensablement progressif du port de Bruges posait problème, mais si les acteurs du commerce au loin privilégièrent Anvers, c'est avant tout en raison d'une attitude beaucoup plus souple et ouverte des autorités anversoises à l'égard des impératifs et besoins commerciaux.
}

19 Plus précisément, le grand commerce échappait à la réglementation courante des échanges. Et lorsqu'il faisait l'objet d'une réglementation spécifique, celle-ci résultait d'un compromis entre les intérêts des hommes d'affaires et ceux des autorités administratives. Bien sûr, là où l'élite des affaires et l'élite politique se confondaient (au sein des cités-États en particulier), cette réglementation spécifique était bien peu contraignante. Mais, même au sein des États-nations en gestation où les autorités administratives avaient des intérêts propres, la réglementation visait plus à articuler la dynamique politique et celle de l'accumulation, qu'à contraindre cette dernière. (Voir en particulier le cas de l'Espagne au XVI ${ }^{\text {ème }}$ siècle.) 
par le déploiement de moyens considérables visant à mettre en œuvre deux stratégies non-disjointes : le monopole ou l'élimination de la concurrence, et la vitesse relative ou le devancement de la concurrence.

\section{Monopoliser l'offre}

Comme on l'a vu précédemment, l'exploitation commerciale des écarts de prix générés par le cloisonnement des marchés exigeait la mobilisation d'investissements et de moyens de toutes sortes extrêmement importants. Les droits d'entrée pesant sur ces activités étaient donc élevés, le degré potentiel de concurrence a priori plutôt faible, et la tendance à la réduction des écarts de prix modérée. Chaque homme d'affaires, ou chaque groupe d'hommes d'affaires, ne s'en efforçait pas moins systématiquement de limiter, autant que faire se peut, le nombre des concurrents susceptibles d'intervenir sur le ou les domaines où se concentraient ses activités, afin de maintenir voire d'accroître les écarts de prix, et de pérenniser les rentes monopolistiques.

\section{$\underline{\text { Monopoles "de fait" }}$}

Tout d'abord, les hommes d'affaires de la Renaissance parvinrent fréquemment à constituer et à maintenir quelque temps des monopoles de fait, c'est-à-dire, à contenir la concurrence par leurs propres moyens, sans le secours des autorités administratives.

Dans cette perspective, la solution la plus évidente pour profiter, ne serait-ce que temporairement, de tel ou tel trafic ou opération commerciale, à l'abri de toute concurrence, était d'être le(s) premier(s) à en organiser matériellement l'exploitation ; une prime était ainsi offerte à l'innovation, à l'initiative et à l'adaptabilité des hommes d'affaires. II ne s'agit cependant là que d'un aspect très particulier, voire ponctuel, de la constitution de monopoles de fait ; d'une part parce que l'innovation commerciale ne garantissait, en tant que telle, qu'un monopole temporaire, et souvent d'autant plus bref qu'il se révélait profitable; d'autre part parce que la stratégie monopolistique des hommes d'affaires ne se limitait évidemment pas aux nouvelles opportunités d'accumulation, et portait tout autant sur les opérations et trafics existants.

De façon plus générale, pour accéder à une position monopolistique et s'y maintenir, pour éliminer ou endiguer la concurrence, les hommes d'affaires de la Renaissance développèrent différents types de pratiques, d'instruments, de relations, et d'institutions, qu'ils combinaient dans des proportions variables selon les spécificités matérielles propres à chacun des "filons" qu'ils entendaient se "réserver".

Comme, dans un nombre croissant de cas, constituer et garantir un monopole de fait dépassait largement les capacités des agents individuels, ceux-ci, hommes d'affaires ou sociétés, furent conduits à se regrouper et à collaborer, afin d'atteindre la masse critique nécessaire pour contrôler l'ensemble d'un domaine d'activités. La plupart du temps, ces regroupements, qu'on qualifierait aujourd'hui d'ententes ou de collusions, revêtaient un fort caractère "national", ce terme devant cependant être entendu dans son acception la plus large, puisqu'il qualifie aussi bien des groupes d'agents originaires d'un même État-nation (Espagnols, Portugais...), d'une même cité (Génois, Vénitiens...), ou même d'entités moins directement définissables (Allemands, Marannes...). ${ }^{20}$

20 Sur le plan institutionnel, ces collaborations étaient, le plus souvent, relativement informelles, à l'image du quasi-monopole qu' exerçaient les Marranes portugais sur le commerce des pierres précieuses au début du XVI ${ }^{\text {ème }}$ siècle. Alors très dispersés géographiquement, habitués à vivre dans le secret, voire dans une clandestinité relative vis à vis du monde qui les entourait, et liés de ce fait par des relations de confiance aussi étroites que discrètes, les Marranes portugais constituaient un réseau international, certes très informel, mais aussi très solide et particulièrement efficace ; présents au Portugal, source quasi-exclusive des pierres précieuses d'Afrique et des Indes, aux Pays-Bas, 
Afin de saisir chaque fois que possible les opportunités d'établir un monopole de fait, ces groupes d'hommes d'affaires recoururent, en fonction des spécificités concrètes de chaque cas, à différentes stratégies, leur permettant de contourner la concurrence et de contrôler l'offre. Ainsi, bien que l'attrait des hommes d'affaires pour la terre ait, le plus souvent, été lié à leur désir de promotion sociale, la propriété foncière fut parfois pour eux un instrument particulièrement efficace de monopolisation de l'offre ; ce fut le cas dans le domaine minier, mais aussi pour quelques produits issus de l'agriculture, et dont les aires de productions étaient étroitement limitées. ${ }^{21}$

Plus massif et plus fréquent fut le recours au crédit, en particulier sous la forme d'achats à terme, qui permettaient simultanément de contrôler une part importante voire la totalité d'une production, et, par la dépendance des producteurs, de garantir la pérennité de ce contrôle. ${ }^{22}$ En la matière, le système le plus abouti, quelque peu postérieur à la Renaissance, fut celui que mirent en place les hommes d'affaires hollandais à partir du début du XVIIème siècle. Grâce à une armée d'agents sillonnant tous les pays où existaient des opportunités afin de traiter directement avec les producteurs, et par le recours systématique à des avances ou à des achats à terme massifs, ils réussirent à s'assurer des positions monopolistiques, ou pour le moins largement dominantes, dans la commercialisation, à l'échelle internationale, de bien des produits : étoffes anglaises, vins français, laine espagnole, cuivre suédois, salpêtre polonais, fourrures russes, cacao du Venezuela, tabac du Maryland...

Une fois établis les monopoles ou quasi-monopoles de fait, le principal souci des hommes d'affaires était de les faire durer, c'est-à-dire de les défendre contre toute velléité concurrentielle. Ces positions monopolistiques ne bénéficiant ni de garantie ni de protection administratives, le comportement le plus fréquemment adopté pour étouffer dans l'œuf toute tentative de remise en cause fut la guerre des prix. ${ }^{23}$

cœur commercial de l'économie-monde occidentale de l'époque, ainsi que sur bien d'autres places majeures, ils firent leur chasse gardée du commerce des pierres précieuses, dont le volume extrêmement faible au regard des valeurs concernées convenait parfaitement à leur situation.

Cependant, les collaborations ou ententes qu'exigeait la mise en œuvre de la stratégie monopolistique se révélèrent parfois plus formelles ou plus organisées, comme le cartel mis en place en 1437 par neuf hommes d'affaires génois pour le contrôle de l'alun, ou celui que constituèrent, en 1498, les principaux exploitants du cuivre tyrolien, en vue de garantir un prix élevé aux livraisons qu'ils effectuaient sur la place vénitienne.

21 Par exemple, la propriété des "terres à soie" fut, à la fin du XV| ${ }^{\text {ème }}$ siècle, le principal instrument du quasi-monopole qu'imposèrent les Génois sur la soie sicilienne, seule alternative aux importations du Levant, et que convoitaient donc bien des hommes d'affaires italiens et européens. Même sans disposer de la totalité des terres et de la production, les hommes d'affaires génois acquirent collectivement un poids suffisant pour que la coordination de leurs comportements leur permette de contrôler les prix de l'ensemble de la soie sicilienne, ou pour mieux dire de les faire. En outre, la propriété foncière leur garantissant les droits de citoyenneté à Messine, ils se trouvaient exonérés des droits d'exportation, ce qui constituait, par rapport à d'éventuels concurrents "étrangers", un avantage important, voire décisif, en matière de commercialisation.

Voir : BRAUDEL, F. Civilisation matérielle, économie et capitalisme. Op cit. Tome 2, pp 370-371.

22 Ainsi, concernant la soie sicilienne, les Génois qui ne contrôlaient directement qu'une partie de la production, procédèrent à des achats à terme massifs aux producteurs siciliens, et parvinrent à empêcher cette part de la production de parvenir directement sur le marché et d'influer sur les prix.

23 C'est ainsi que les Génois, confrontés à une tentative de grande envergure menée par des marchands soyeux de Tours décidés à briser leur monopole sur la soie sicilienne, réagirent en commercialisant immédiatement, sur la place lyonnaise, d'importantes quantités de soie à un prix sensiblement inférieur à celui que les Tourangeaux acquittaient, en Sicile, pour leur approvisionnement. On peut également citer la guerre des prix du cuivre au début du XV ${ }^{\text {ème }}$ siècle, cas un peu particulier, puisqu'elle opposa deux "monopoles", et que son acteur majeur avait des 
On constate ainsi que les hommes d'affaires de la Renaissance mobilisèrent des fonds importants, développèrent des formes variées de collaboration, et mirent en œuvre toutes sortes d'instruments et de pratiques, afin d'établir et de préserver le plus longtemps possible des monopoles ou quasi-monopoles de fait, c'est-à-dire, afin d'échapper à la concurrence, de peser sur la détermination des prix et d'engranger les rentes monopolistiques. Cependant, quels qu'aient été les efforts déployés pour les préserver, les positions monopolistiques de fait et les profits qu'elles engendraient étaient, par nature, susceptibles d'être remis en question à tout moment par de nouveaux concurrents. Dans cette perspective, et malgré leur réticence générale à l'égard de la réglementation des activités commerciales, les hommes d'affaires prirent très tôt conscience de ce que, pour établir et préserver des positions monopolistiques, les autorités administratives pouvaient leur être d'un grand secours, d'une part parce qu'elles étaient en mesure de leur accorder des avantages décisifs sur leurs éventuels concurrents, d'autre part et surtout, parce qu'elles seules avaient le pouvoir d'interdire purement et simplement la concurrence, et de faire respecter de telles interdictions.

\section{$\underline{\text { Monopoles "administratifs" }}$}

C'est pourquoi, au-delà des moyens déjà évoqués, les relations avec les autorités administratives furent au cœur de la mise en œuvre de la stratégie monopolistique des hommes d'affaires, ou tout au moins, des plus importants d'entre eux. En effet, les interventions administratives en faveur des monopoles n'avaient rien de gratuit ; pour traiter avec les autorités et en obtenir garanties et protections, les hommes d'affaires se devaient d'être en mesure de proposer des contreparties motivantes. Les autorités administratives de l'époque ayant fréquemment d'importants besoins financiers, ces contreparties prenaient avant tout la forme de crédits accordés aux princes, auxquels s'ajoutaient parfois des fournitures de marchandises (en particulier pour l'équipement et le ravitaillement des armées), une aide dans la gestion de leurs biens personnels ou de leurs revenus fiscaux, ou, plus simplement, une part des profits générés par leur intervention.

En réponse aux facilités financières consenties et aux divers services rendus, les hommes d'affaires pouvaient tout d'abord espérer obtenir des privilèges fiscaux ou douaniers leur assurant un avantage concurrentiel à partir duquel il était plus aisé d'accéder à une position monopolistique. ${ }^{24}$

Ces privilèges, qui facilitaient les monopoles de fait et en renforçait la durabilité, ne constituaient cependant que le premier degré de ce que les hommes d'affaires pouvaient attendre des relations, en particulier financières, qu'ils entretenaient avec les autorités administratives. En la matière,

intérêts dans les deux camps : Jacob Fugger, qui venait d'obtenir, pour sa seule société, un monopole administratif sur le cuivre hongrois, parvint à ruiner le cartel du cuivre tyrolien, dont il avait pourtant été l'initiateur.

Voir : MARGOLIN, J-C. L'avènement des Temps Modernes. Paris. P.U.F. 1977. p 92.

${ }^{24}$ Ainsi, dès le XIV ${ }^{\text {ème }}$ siècle, de très grandes maisons florentines, telles celle des Bardi ou celle des Peruzzi, se virent accorder des franchises fiscales en Angleterre et furent exonérés de droits de douane sur les exportations de laine anglaise, en contrepartie de crédits énormes accordés aux souverains ; de façon similaire, elles obtinrent du roi de Sicile des franchises douanières sur les exportations de céréales. À la même époque, l'une des plus riches familles d'affaires vénitiennes, les Corner, s'empressant de répondre aux besoins financiers des rois de Chypre, put en échange, et au grand dam des autres négociants vénitiens, disposer d'un contrôle de fait sur le coton, le sucre et le sel chypriotes. De même, les dispenses et exonérations obtenues par Jacques Cœur en contrepartie des innombrables services de toutes sortes qu'il rendit à Charles VII, furent pour beaucoup dans le quasi-monopole qu'il parvint à établir, à la fin de la première moitié du XV ${ }^{\text {ème }}$ siècle, sur les échanges commerciaux entre le Royaume de France et l'Orient. 
le summum était le "monopole de droit", officiellement accordé par les autorités administratives, parfois moyennant rémunération, et que celles-ci prenaient sur elles de faire respecter. ${ }^{25}$

Ainsi, au-delà de tout ce que les hommes d'affaires de la Renaissance entreprirent pour accéder, par eux-mêmes, à des positions de monopole, l'établissement de relations privilégiées avec les princes et les autorités administratives fut, pour les plus importants d'entre eux, un élément central de leur stratégie monopolistique. Bien sûr, de leur point de vue ces relations étaient parfois très coûteuses, mais les retours d'investissement pouvaient se révéler considérables, en termes de profits bien sûr, mais aussi en termes de statut social. Dans la mesure où ils permettaient aux hommes d'affaires qui en bénéficiaient de s'affranchir de la concurrence et de bénéficier de rentes monopolistiques, avantages concurrentiels et monopoles de droit accordés par les princes et autorités administratives valaient largement les facilités financières et aides diverses consenties en contrepartie. ${ }^{26}$

\section{Devancer les concurrents}

La stratégie monopolistique mise en œuvre par les hommes d'affaires de la Renaissance répondait au souci d'exploiter, de la façon la plus profitable possible, des opportunités ou des écarts de prix potentiellement durables ; c'était, par nature, une stratégie à moyen ou long terme, dont l'un des objectifs centraux était justement de pérenniser les écarts de prix. Cependant, dans l'économie-monde occidentale de la Renaissance, bien des écarts de prix survenaient soudainement et n'étaient qu'éphémères, bien des opportunités n'étaient que ponctuelles et devaient être saisies rapidement. Dans cette perspective, la stratégie monopoliste était inopérante. Le seul objectif que dictait la logique d'accumulation aux hommes d'affaires était d'agir à temps, c'est-à-dire, avant que l'écart de prix ne disparaisse. On verra que, concrètement, dans la plupart des cas, agir à temps revenait à agir avant les autres, à devancer les concurrents. Pour y parvenir, les hommes d'affaires de la Renaissance déployèrent des efforts considérables, et développèrent des comportements et pratiques totalement

\footnotetext{
25 C'est ainsi que Jacob Fugger qui n'avait jamais compté son soutien financier à Charles Quint reçut en "récompense" le monopole du cuivre hongrois, que l'homme d'affaires marrane Joseph Naci se vit accorder par Soliman le Magnifique le monopole du commerce de vin entre l'Empire Ottoman et I'Europe Centrale, ou que des sociétés siennoises puis génoises bénéficièrent, au XVI ${ }^{\mathrm{me} e}$ siècle, du monopole de l'exploitation des mines d'alun de la papauté. On vit même, parfois, l'attribution de monopoles de droit être l'enjeu d'une concurrence organisée par les autorités administratives ; ainsi la concession, par la Commune de Gênes, du monopole d'exploitation de l'alun de Phocée, faisait-elle l'objet, au XV ${ }^{\text {ème }}$ siècle, d'une adjudication aux enchères.
}

26 On ne saurait traiter, même brièvement, des monopoles de la Renaissance, sans rappeler qu'au $\left.\mathrm{XV}\right|^{\mathrm{eme}}$ siècle, Espagnols et Portugais réalisèrent au moins les trois quarts des trafics intercontinentaux, et sans justifier le fait que cette situation quasi-monopolistique n'ait pas été évoquée. C'est que, au moins jusqu'à la fin du XVI ${ }^{\text {ème }}$ siècle, et même si des hommes d'affaires ibériques en tirèrent d'énormes profits, les quasi-monopoles espagnol et portugais sur le commerce avec les mondes lointains relèvent plus de la gestation d'États-nations impériaux, que des stratégies concurrentielles des hommes d'affaires : au Portugal, l'affrètement des navires et l'ensemble des trafics étaient directement organisés par la Couronne à travers la Casa da Guiné puis la Casa da India ; et en Espagne, la Casa de Contratacion puis le Conseil des Indes, chargés d'administrer et de contrôler le quasi-monopole des échanges avec le Nouveau Monde, avaient comme premier souci de préserver les intérêts de la Couronne.

Bien sûr, et en particulier en Espagne, les hommes d'affaires finiront, à la charnière des $X V I^{e ̀ m e}$ et $X V I I{ }^{e ̀ m e}$ siècles, par faire prévaloir leurs intérêts au sein de ces institutions, tandis que chez les nouveaux acteurs du commerce intercontinental, et en premier lieu aux Pays-Bas, se constitueront des "compagnies nationales" totalement dévolues aux intérêts marchands. 
antinomiques avec les lois de la concurrence parfaite, et en particulier avec celles ayant trait à la transparence de l'information.

\section{Volatilité des prix et stratégies concurrentielles}

D'une façon générale, la volatilité importante de certains prix, dans l'économie-monde occidentale de la Renaissance, résultait de deux phénomènes interdépendants. D'une part, l'irrégularité de l'offre et/ou de la demande sur certains marchés était source de fluctuations de prix, parfois de grande ampleur. C'était particulièrement le cas des marchés relevant du commerce au loin, sur lesquels les quantités échangées étaient relativement faibles, l'offre à l'échelle locale inévitablement irrégulière, et la demande fluctuante au gré des modes. ${ }^{27}$ De façon plus épisodique, les marchés de produits agricoles et en particulier de céréales, infiniment plus importants en termes de quantités échangées, mais dont l'offre était soumise à bien des aléas, (climatiques, militaires...) pouvaient, eux aussi, connaître des fluctuations de prix très importantes, et donc très attractives pour les hommes d'affaires. D'autre part, sur ces marchés sujets à des fluctuations de l'offre et/ou de la demande, la diffusion de "nouvelles" de tous ordres, vraies ou fausses, mais dont le contenu était susceptible d'influer sur les prix, provoquait parfois des comportements de panique et des réactions en chaîne générant des variations de prix soudaines et de grande ampleur, ainsi que des sur-ajustements. ${ }^{28}$

A priori, ces fluctuations importantes recelaient des opportunités d'accumulation alléchantes. Dans le cas d'un écart de prix temporaire entre deux places commerciales distinctes, une opération relevant de l'arbitrage pouvait se révéler particulièrement fructueuse, à condition, bien sûr, qu'elle se réalise avant que l'écart de prix ne diminue ou ne disparaisse. Dans le cas de variations soudaines en un lieu donné, être en mesure d'anticiper les mouvements était la promesse de profits importants et rapides. Dans les deux cas, pour un homme d'affaires, tirer profit de ces écarts et fluctuations de prix supposait d'agir avant les autres, avant que les actions des concurrents ne fassent disparaître les opportunités.

Concrètement, le principal et le plus sûr moyen dont disposait un homme d'affaires pour agir avant ses concurrents, consistait à être informé avant eux des opportunités à saisir. ${ }^{29}$ Plus précisément, il s'agissait, pour chaque homme d'affaires, d'obtenir des informations dont les concurrents ne disposaient pas ou pas encore, et de faire en sorte qu'ils y aient accès le plus tard possible. Bien loin de promouvoir la transparence de l'information, les hommes d'affaires recherchaient donc systématiquement l'antériorité et l'exclusivité, au moins temporaire, de l'information ; il n'est sans doute

27 Par exemple, à la fin du XIV ème siècle, les cours parisiens des fourrures d'Europe orientale, dont le commerce était saisonnier, variaient parfois de plus de $30 \%$ d'une année sur l'autre.

Voir : FAVIER, J. Op cit. p 84.

28 Par exemple, à Venise, le prix du froment augmente de 12,5\% au cours de la seule journée du 3 janvier 1503, car on apprend qu'un convoi de navires, envoyé en Sicile pour y charger des blés, a trouvé les concessions d'exportation suspendues. De même le 5 décembre, la hausse est de $20 \%$, suite à la nouvelle du naufrage d'un navire revenant de Chypre avec une cargaison de blés. On peut noter que le marché des assurances maritimes était tout particulièrement sensible aux nouvelles. Ainsi, le 14 mars 1501, deux nobles vénitiens libérés par les Ottomans arrivèrent à Venise, et annoncèrent qu'une flotte de corsaires turcs d'une quarantaine de navires venait de prendre la mer ; instantanément, les primes d'assurance sur l'aller simple Venise-Beyrouth passèrent de 1,5\% à $10 \%$ de la valeur de la cargaison.

D'après différentes données fournies par : SARDELLA, P. Op cit.

29 «La prime est à celui qui sait avant d'autres - et qui, pendant quelques jours ou quelques heures, est le seul à savoir - ce que sera demain la guerre ou la paix, la disette ou l'abondance.»

Voir : FAVIER, J. Op cit. pp 83-84. 
pas abusif de dire que les hommes d'affaires de la Renaissance érigèrent l'asymétrie d'information et le délit d'initiés en principes.

Bien sûr, mettre en œuvre cette stratégie de devancement de la concurrence par la quête de la vitesse relative de circulation de l'information, exigeait, en premier lieu, d'avoir régulièrement accès à des services postaux très performants, ce qui, comme on l'a vu, n'était pas à la portée du premier négociant venu. Cette condition étant remplie, on pouvait participer à la course à l'information (au moins temporairement) exclusive, course dont l'enjeu conduisit les hommes d'affaires de la Renaissance à développer des pratiques et moyens entrant fréquemment en contradiction frontale avec les lois théoriques du marché.

\section{L'organisation matérielle du devancement de la concurrence}

Pour disposer, avant les autres, d'informations sur les opportunités temporaires d'accumulation, ou de nouvelles susceptibles de provoquer des variations de prix significatives, le moyen le plus évident résidait dans un accès privilégié aux différentes sources d'informations. Dans cette perspective, l'avantage était à celui qui avait accès au plus grand nombre d'informateurs ou de correspondants, et au réseau le plus étendu et le plus ramifié.

En ce qui concerne plus spécifiquement les nouvelles ayant trait aux évènements d'ordre politique ou aux décisions des autorités administratives, les relations très étroites qu'entretenaient certains hommes d'affaires avec les princes et gouvernants, quand ils ne gouvernaient pas eux-mêmes, constituaient un atout de choix. Là où le pouvoir politique était entre les mains d'une oligarchie aristomarchande, l'exercice de charges publiques pouvait se révéler extrêmement fructueux. ${ }^{30}$

Aux efforts déployés par chaque homme d'affaires d'envergure pour constituer un réseau d'informateurs lui permettant d'accéder plus directement et plus rapidement que ses concurrents aux différentes sources d'information, s'ajoutait un certain nombre de pratiques visant très clairement à entraver l'accès des concurrents à l'information. D'une part, il va sans dire que lorsqu'un homme d'affaires disposait d'une information laissant entrevoir la possibilité d'opérations particulièrement rentables, à condition d'agir seul, il se gardait bien de la transmettre à ses confrères. Plus généralement, dès lors que la divulgation d'une information était de nature à remettre en cause, ne serait-ce que partiellement, les perspectives d'accumulation dont elle était porteuse, la confidentialité était de règle ; secrets et mensonges, au moins par omission, étaient ainsi une caractéristique primordiale des relations entre hommes d'affaires concurrents, et un obstacle majeur à une parfaite circulation de l'information.

D'autre part, ceux des hommes d'affaires auxquels l'organisation matérielle de la circulation de l'information en offrait la possibilité, ne se privaient pas de retarder, délibérément, l'accès de leurs concurrents aux informations qui leur étaient destinées. On peut souligner que ces actions délibérées d'entrave au fonctionnement harmonieux du marché apparaissaient si "normales" aux hommes

30 Par exemple, à Venise, où le rôle économique de l'État était si important, les familles d'affaires représentées au Sénat bénéficiaient d'un accès privilégié à bien des informations cruciales pour la vie des affaires. Et là où le pouvoir des princes demeurait prépondérant, crédits, conseils et services divers étaient, pour les hommes d'affaires qui en avaient les capacités, autant de moyens de se rapprocher, au dépens de leurs concurrents, de cette source d'informations essentielle du point de vue de l'accumulation. L'exemple des Médicis au début de la seconde moitié du XV ${ }^{\text {ème }}$ siècle est, à cet égard, particulièrement éclairant : s'ils étaient, de fait, les souverains de Florence, berceau de leur maison commerciale, Bruges, où ils avaient installé l'une de leurs principales filiales, était alors sous le contrôle des Ducs de Bourgogne ; cela n'empêcha aucunement les Médicis d'y bénéficier d'une priorité en matière d'informations d'ordre politique, puisque le directeur de la filiale brugeoise, Tommaso Portinari, fut un bailleur de fonds et un conseiller influent de Philippe le Bon, puis de Charles le Téméraire. 
d'affaires, qu'elles se faisaient au grand jour ; ainsi était-il de notoriété publique que la Scarcella, l'entreprise postale florentine, distribuait le courrier dont elle avait la charge de façon discriminatoire ; lorsqu'un paquet de lettres arrivait à destination, celles destinées à des associés de l'entreprise étaient distribuées un ou deux jours avant celles des autres clients. ${ }^{31}$ D'ailleurs, dès la seconde moitié du XIVème siècle, le fait d'entraver l'accès de ses concurrents à l'information était présenté comme une règle professionnelle de bon sens, dans un traité de pratiques commerciales rédigé par l'homme d'affaires italien Paolo de Certaldo ${ }^{32}$ :

« Si tu exerces le commerce, et qu'avec la correspondance de ta maison, on te remet des lettres pour d'autres destinataires, aie toujours soin de lire d'abord les tiennes, avant de distribuer aux autres les leurs ; et si tes lettres te suggèrent d'acheter ou de vendre telles marchandises pour en tirer bénéfice, cherche immédiatement un courtier, et fais ce que ces lettres te disent ; ensuite seulement, distribue les lettres arrivées avec les tiennes.»

Enfin, ce qui symbolise le mieux l'importance des moyens mis en œuvre par les hommes d'affaires de la Renaissance afin de devancer la concurrence est, sans doute, le prix qu'ils étaient prêts à payer pour qu'une information leur apparaissant cruciale circule plus rapidement pour eux que pour leurs concurrents, c'est-à-dire pour profiter d'une exclusivité au moins temporaire de l'information. Les exemples sont multiples. ${ }^{33}$ On se contentera de remarquer qu'à Venise, au début du XVIème siècle, le prix de la transmission d'une lettre à Rome par le service le plus rapide (environ 40 heures), représentait près de 3,5 fois les revenus annuels d'un ouvrier terrassier, et près du quart des revenus annuels du directeur de la perception des douanes ; quant à l'affrètement d'un bateau-courrier express pour Beyrouth, les rapports respectifs étaient de 60 à 70 et de 4 à $5 .{ }^{34}$

Du fait des coûts exponentiels de la vitesse de transmission des nouvelles, la vitesse d'accès des agents à l'information était profondément inégale, et c'est de cette inégalité que découlaient les opportunités éphémères. Seuls les hommes d'affaires de très grande envergure disposaient des moyens nécessaires pour participer à la course aux profits exceptionnels qu'autorisait le devancement de la concurrence. Et cette course, entre hommes d'affaires capables d'en acquitter les droits d'entrée, donnait lieu à toutes sortes de comportements et pratiques pour le moins éloignés des principes de l'information et de la concurrence parfaites. ${ }^{35}$

31 Voir : FAVIER, J. Op cit. p 87.

32 Cité par : SAPORI, A. Le marchand italien du Moyen Age. Paris. Armand Colin. 1952. p 37.

33 Par exemple, en octobre 1338, la maison Peruzzi de Florence, craignant que le revers subi par Edouard III en Flandre n'inquiète ses déposants et ne la mette en difficulté du fait de ses importants engagements financiers auprès de la Couronne anglaise, n'hésita pas à affréter spécialement un bateau et à mandater un messager pour avertir sa filiale de Rhodes, de façon à ce que celle-ci puisse prendre quelques mesures préventives avant que la nouvelle ne soit là-bas publique ; on imagine sans peine les frais engendrés par une telle décision. Et le recours aux entreprises spécialisées n'était pas forcément moins onéreux ; ainsi, à la même époque, le prix des services postaux florentins les plus rapides était, pour un trajet tel que Florence-Avignon, d'environ 15 florins, soit près de 60 grammes d'or. Voir : RENOUARD, Y. Op cit. p 137.

${ }^{34}$ D'après des données fournies par : SARDELLA, P. Op cit. pp 50-51.

35 C'est, sans doute, au cours de la seconde moitié du XV ${ }^{\text {ème }}$ et au début du XVI ${ }^{\text {ème }}$ siècles, que la lutte sans merci à laquelle se livraient les hommes d'affaires importants, dans le cadre de leur stratégie individuelle de devancement de la concurrence, fut la plus vive, et la source des dérives les plus importantes du point de vue des lois théoriques du marché. II convient cependant de souligner que si cette lutte perdit quelque peu de son intensité par la suite, tandis que se renforçait la tendance monopolistique, les hommes d'affaires n'en continuèrent pas moins d'y consacrer des moyens significatifs, comme le montre le système d'embarcations légères rapides mis en place en Mer du 
Finalement, qu'il s'agisse de s'affranchir du cloisonnement caractéristique des marchés de l'époque, d'entraver, d'éliminer ou de devancer la concurrence, les stratégies que leur dictait la logique d'accumulation conduisirent les hommes d'affaires de la Renaissance à élaborer et à développer des comportements, des pratiques, des instruments, des techniques, des conventions et des institutions, leur permettant, pour leur plus grand profit, d'opérer en marge des lois, tant théoriques qu'historiques, du marché.

\section{Capitalisme et Marché}

Ce rapide survol des stratégies, pratiques et comportements concurrentiels développés par les hommes d'affaires de la Renaissance, met en lumière les principaux traits de l'articulation historique du marché et du capitalisme, à l'époque où celui-ci émergeait.

\section{À la Renaissance}

En rupture avec l'attitude traditionnelle des marchands à l'égard des contraintes de tous ordres pesant sur l'organisation matérielle des échanges, ${ }^{36}$ les hommes d'affaires de la Renaissance s'efforcèrent, sans relâche et par tous les moyens, de faire en sorte que leurs activités échappent à l'emprise des lois historiques du marché. Ils parvinrent ainsi à développer leurs activités dans un "univers" commercial distinct de celui que définissaient les lois historiques; dans la mesure où la première caractéristique de cet univers était d'échapper au cloisonnement spatial, on peut le désigner par le terme de marché global. C'est ainsi qu'aux côtés des structures de marché héritées de l'Expansion Médiévale, et qui continuaient d'encadrer la plus grande masse des échanges, les hommes d'affaires promurent l'essor de ce marché global, en mettant en œuvre des pratiques et des principes de fonctionnement se différenciant de plus en plus nettement de ceux du reste de la classe marchande.

Outre leur spécificité historique, ces pratiques et principes de fonctionnement apparaissent non seulement distincts, mais en outre clairement contradictoires avec ceux censés garantir une allocation optimale des ressources. ${ }^{37}$ D'une part, le marché global promu par les hommes d'affaires se caractérisait par un nombre d'acteurs très réduit; du fait de l'envergure des opérations qui s'y déroulaient, le niveau des droits d'entrée à acquitter pour y accéder était absolument inaccessible à la presque totalité de la classe marchande. D'autre part, si les formes concrètes de la concurrence entre hommes d'affaires rompaient avec l'"égalitarisme" très encadré que les autorités administratives faisaient régner sur les marchés traditionnels, elles se traduisaient par des stratégies monopolistiques,

Nord par les spéculateurs d'Amsterdam, à la fin du XVIII ${ }^{\text {ème }}$ siècle. Voir : BRAUDEL, F. Civilisation matérielle, économie et capitalisme. Op cit. Tome 2, p 362.

36 Sur cette attitude générale des hommes d'affaires de la Renaissance, au delà des contraintes administratives, spatiales et techniques ici évoquées, voir : WALERY, S. Les hommes d'affaires de la Renaissance et l'"esprit" du capitalisme : l'exemple de la lettre de change. Actes du Forum de la Régulation 2003.

37 Sur un plan logique, la distinction capitalisme/lois théoriques du marché est un truisme, puisque ces deux concepts visent à rendre compte de deux logiques différentes ; ce que s'efforçaient de promouvoir et de développer les hommes d'affaires de la Renaissance, ce n'est évidemment pas l'allocation optimale des ressources, mais l'accumulation du capital. En outre, le concept de capitalisme étant, par nature, historique, et les lois théoriques du marché a-historiques, leur assimilation ne peut résulter que d'une confusion extrême. 
ententes, collusions, secrets et pratiques déloyales, encore plus antinomiques, en particulier en matière d'information, avec les conditions qu'exige la concurrence parfaite. ${ }^{38}$

C'est cependant sur la base de ces pratiques et principes de fonctionnement, que les hommes d'affaires de la Renaissance parvinrent à tirer profit de leur position d'autonomie vis à vis du cadre historique du marché, pour en exploiter systématiquement les faiblesses. Le plus souvent, la profitabilité des opérations projetées et réalisées par les hommes d'affaires découlait en effet directement des possibilités qu'offrait le marché global d'exploiter certaines situations inhérentes au cadre traditionnel des échanges, et ce, de façon d'autant plus rentable que les volumes traités et le nombre des acteurs intervenant sur ce marché demeuraient faibles au regard de ceux des marchés traditionnels. C'est ainsi que les opérations que privilégiaient les hommes d'affaires consistaient à exploiter, tantôt le cloisonnement géographique et la variabilité spatiale des prix propres aux marchés traditionnels, tantôt les faiblesses et les imperfections de la circulation de l'information, tantôt le morcellement monétaire et le caractère embryonnaire des structures de financement, tantôt les multiples entraves réglementaires pesant sur l'organisation des activités marchandes courantes... Dans la plupart des cas, le profit et l'accumulation trouvaient leur source dans l'exploitation de ce qu'on pourrait appeler, du point de vue des lois théoriques du marché, les imperfections du cadre que définissaient les lois historiques, les imperfections du "marché historique".

Finalement, dans les conditions historiques de la Renaissance, l'accumulation du capital, mise en œuvre par les hommes d'affaires, s'est développée à travers l'exploitation, fondée sur des principes antinomiques avec les lois théoriques du marché, d'une position d'autonomie à l'égard des lois historiques continuant de s'imposer à la masse des échanges et des marchands. Au moment de son émergence, sous sa forme commerciale, le capitalisme s'est ainsi développé en se plaçant en marge des lois historiques et théoriques du marché, afin de mieux tirer profit des imperfections de celui-ci. En s'affranchissant progressivement des lois historiques, le capitalisme commercial se constitue en "organisme" distinct du marché de la Renaissance ; en tirant profit des situations générées par le fait que la quasi-totalité des échanges continuaient, eux, de s'inscrire dans le cadre des lois historiques, le capitalisme commercial se constitue en organisme parasite ${ }^{39}$ du marché de la Renaissance.

Comme l'a si souvent répété Fernand Braudel, ${ }^{40}$ la claire distinction du capitalisme et du marché est ainsi un préalable indispensable à toute appréhension cohérente de l'émergence du capitalisme. Comment appréhender rigoureusement un organisme parasite sans le distinguer de l'organisme qu'il parasite? Appliquée aux conditions historiques de la Renaissance, l'assimilation du capitalisme et du marché est une aberration logique qui interdit de penser l'émergence du capitalisme.

38 II existe entre l'accumulation commerciale mise en oeuvre par les hommes d'affaires de la Renaissance et les lois théoriques du marché, une contradiction irréductible qui découle tout simplement de ce que, dans un univers conforme aux lois théoriques du marché, l'accumulation ne peut être réalisée par, ou à travers, l'échange.

39 Parasitisme : association de deux organismes [vivants], l'un vivant aux dépends de l'autre, mais sans le détruire.

40 Ainsi notait-il, dans la conclusion de Civilisation matérielle, économie et capitalisme (Op cit. Tome $3, \mathrm{p} 538)$ :

«(...) le capitalisme distinct de l'économie de marché, pour moi, le témoignage essentiel de ma longue recherche.» 


\section{Les échos contemporains de l'assimilation du capitalisme et du marché}

Les relations du capitalisme avec les lois historiques et avec les lois théoriques du marché sont, en tant que fait historique, variables. " II n'y a d'immuable que l'abstraction du mouvement ».41 Elles n'ont, depuis la Renaissance, cessé de se transformer.

Outre le fait que, par nature, les lois historiques du marché sont évolutives, l'essor du capitalisme, et les très profondes mutations qu'il a traversées depuis la Renaissance, ont conduit à de multiples redéfinitions de l'articulation du marché historique et du capitalisme. Se sont ainsi succédées des périodes durant lesquelles la dynamique du capitalisme paraît avoir lourdement pesé sur les lois historiques du marché,42 et des périodes durant lesquelles elle dut, visiblement, composer avec d'autres dynamiques. ${ }^{43}$ L'articulation "parasitaire" du capitalisme et du marché historique, caractéristique de la Renaissance, ne doit donc pas faire l'objet d'extrapolations a-historiques.

II importe pareillement de se garder de toute dérive tendant à attribuer une portée universelle aux formes de la contradiction qu'on a pu observer entre le capitalisme commercial des origines et les lois théoriques du marché. Pour autant une telle contradiction ne saurait non plus être considérée comme une exception historique, exclusivement liée aux spécificités du capitalisme commercial de la Renaissance ; par exemple, la mutation que connut le capitalisme à la fin du XIXème et au début du $X X$ ème siècle, et qui se traduisit, entre autres, par une forte concentration du capital ${ }^{44}$ et une régression sensible de l'atomicité du marché du travail, apparaît, elle aussi, quelque peu antinomique avec les lois théoriques du marché.

Au delà de ces indispensables précautions, de longs développements sont sans doute superflus, pour percevoir les échos contemporains de ce qui vient d'être dit des relations du capitalisme et du marché à la Renaissance, pour constater que l'assimilation du capitalisme et du marché n'est pas moins inopérante ni invalidante, appliquée à la période contemporaine, qu'elle ne le serait, appliquée à la Renaissance et au capitalisme commercial. II est ainsi d'une grande banalité de constater que les entreprises et firmes contemporaines s'efforcent, tout autant que les hommes d'affaires de la Renaissance, de développer leurs activités en marge des lois théoriques du marché. « Ce ne sont pas les entreprises qui maintiennent la concurrence, au contraire, elles visent toutes à l'entente, à l'oligopole, à la domination du marché (...). $»^{45}$ Parallèlement, la période récente a clairement montré à quel point les lois historiques du marché forgées à l'époque du fordisme constituent, depuis les années 1970, autant de contraintes pesant sur l'accumulation, que le développement capitaliste contemporain tend à élargir, voire à supprimer. Bref, capitalisme et marché, que celui-ci soit appréhendé dans sa dimension "théorique" ou dans sa dimension "historique", ne sont pas moins distincts aujourd'hui qu'ils ne l'étaient à la Renaissance.

41 Voir : MARX, K. Misère de la philosophie. Paris. Éditions Sociales. 1972. p 119.

42 La disparition plus ou moins brutale des systèmes corporatifs face à l'essor du capitalisme industriel, à la fin du XVIII ${ }^{\mathrm{eme}}$ et au début du XIX ${ }^{\text {eme }}$ siècle ; l'ensemble des mesures récentes ayant trait à la concurrence ou au marché du travail, et qu'on synthétise couramment par le terme de "déréglementation"...

43 La montée en puissance des États-nations mercantilistes au XVII ${ }^{\text {ème }}$ et au début du XVIII ${ }^{\text {ème }}$ siècle ; la montée en puissance de l'intervention publique et des protections et garanties pour le salariat, au lendemain de la seconde guerre mondiale...

44 Plus particulièrement aux États-Unis et en Allemagne.

45 Voir : BOYER, R. Une théorie du capitalisme est-elle possible ? Paris. Odile Jacob. 2004. p 11. 
Pourtant, bien que logiquement et concrètement aberrante, l'assimilation du capitalisme et du marché, et tout particulièrement l'assimilation du capitalisme et des lois théoriques du marché, sont, aujourd'hui plus que jamais, des lieux communs ${ }^{46}$ largement répandus. Qui plus est, cette assimilation parvient à réconcilier des points de vue par ailleurs violemment opposés : elle est, par exemple, un point de convergence implicite entre des responsables d'organisations professionnelles aussi différents qu'Ernest-Antoine Seillière, l'ancien Président du Medef, et José Bové, l'ancien Porte-Parole de la Confédération Paysanne.

De la même façon, elle est, dans le champ de l'économie, un des très rares points de convergence implicite entre les purs Libéraux, pour lesquels ce que l'on appelle le capitalisme n'est rien d'autre que le Marché, et les plus radicaux, pour lesquels ce que l'on appelle le marché ou l'économie de marché n'est rien d'autre que le Capitalisme. Le plus étonnant est que, même des économistes s'efforçant de tracer une voie médiane, tel Jean Paul Fitoussi, dans La démocratie et le marché, reprennent à leur compte cette confusion. Au moins celui-ci a-t-il le mérite de fournir une rapide explication :

«La thèse libérale (...) est fondée sur la théorie (...) de l'équilibre général de concurrence parfaite. Ce sont ses résultats qui sont le plus souvent invoqués en défense et illustration du capitalisme. ${ }^{47}$

Doit-on en conclure que capitalisme et marché sont une seule et même chose, parce que les thuriféraires du capitalisme invoquent les résultats de la théorie de Walras ? Et ce, alors que cette théorie ne prétend pas rendre compte d'une réalité mais caractériser un idéal abstrait impossible à atteindre, et que les acteurs principaux du capitalisme, entrepreneurs, firmes et détenteurs de capitaux, ne prospèrent qu'en contournant les principes de ladite théorie. Par égard pour l'auteur, on se gardera de qualifier la cohérence logique de son propos.

\section{Une mystification idéologique, dernier avatar de la négation de l'histoire}

Comme le soulignait Fernand Braudel en son temps, ${ }^{48}$ l'imbroglio logique et théorique que constitue l'assimilation du capitalisme et du marché a pris sa source dans la confrontation du monde soviétique et du monde capitaliste, qui a marqué le XXème siècle ${ }^{49}$; il est un héritage de la révolution russe et de la guerre froide. Selon la propagande soviétique, toute compromission avec le marché revenait à mettre un doigt dans l'engrenage infernal du capitalisme ; selon la propagande capitaliste, toute mise en cause réelle de la dynamique du capitalisme mettait en danger la liberté du marché, et constituait ainsi le premier pas vers le totalitarisme. On comprend aisément que, dans ce contexte de confrontation stratégique, l'assimilation du marché et du capitalisme relevait des intérêts communs aux deux adversaires : interdire l'émergence de toute alternative aux deux modèles existants.

La disparition du monde soviétique et la disqualification du modèle de planification centralisée, conjuguées au renouveau planétaire de l'influence libérale, se sont traduits à la fois par un renforcement et par un certain "glissement" de cette assimilation. Comme le souligne implicitement Jean Paul Fitoussi dans la précédente citation, le néo-libéralisme entretient très activement la confusion du capitalisme et des seules lois théoriques du marché : la propagande des thuriféraires actuels du

\footnotetext{
46 Lieu commun : thèse à partir de laquelle on argumente, mais sur laquelle on n'argumente pas.

47 FITOUSSI, J-P. Op cit. p 52. C'est nous qui soulignons.

48 Voir, en particulier : BRAUDEL, F. La dynamique du capitalisme. Paris. Arthaud. 1985. pp 118-119.

49 On notera que cette assimilation n'est présente ni chez Marx (il y a une différence de nature entre le cycle de la marchandise et celui du capital), ni chez Walras, incarnation du marché et socialiste, au sens que revêtait ce terme à la fin du XIX ${ }^{\text {ème }}$ siècle.
} 
capitalisme consiste à affirmer que les évolutions et réformes qu'ils promeuvent ne visent qu'à rendre plus efficace le fonctionnement du marché, en s'appuyant sur ses lois théoriques immuables.

Cette propagande repose sur une véritable mystification. Qui peut croire un seul instant que l'univers qu'appellent de leur vœux les laudateurs contemporains du capitalisme ressemble, même de très loin, à l'univers walrassien, à l'utopie du marché de concurrence parfaite ? Qui peut croire que les promoteurs du capitalisme contemporain rêvent de marchés atomisés parfaitement concurrentiels, de rendements décroissants, de transparence de l'information, d'un mode de coordination des choix individuels totalement centralisé, 50 et par dessus tout, de l'absence de profit entrepreneurial, caractéristique de l'équilibre général?

Cette mystification n'est que la traduction contemporaine d'une tentative idéologique récurrente des promoteurs du capitalisme ; elle est le dernier avatar ${ }^{51}$ de la négation de l'histoire. L'assimilation du capitalisme et du marché a ainsi pour première finalité de faire passer les lois historiques favorables au développement capitaliste pour des principes universels a-historiques, dictés par la seule raison. II s'agit, aujourd'hui comme hier, de faire du capitalisme un horizon a-historique, un horizon indépassable.

Comme si le marché contemporain n'était pas régenté par des lois historiques largement déterminées par les impératifs de l'accumulation, comme si les acteurs du capitalisme n'étaient pas prêts à promouvoir d'autres lois historiques dès lors que les conditions et impératifs de l'accumulation viendraient à se modifier, comme si les évolutions que les promoteurs du capitalisme appellent de leurs vœux ne relevaient pas de l'histoire. Bref, comme si le marché n'était pas un mode d'allocation des ressources dont les résultats concrets sont très largement conditionnés par les lois historiques qui l'instituent et le régentent. Et comme si le capitalisme avait jamais promu les lois théoriques du marché, lui qui n'a comme seule préoccupation que de promouvoir les lois historiques qui, dans les conditions de l'époque, permettent (parfois en y dérogeant) le rythme d'accumulation le plus élevé possible.

La négation de l'histoire que les promoteurs actuels du capitalisme propagent avec ferveur en assimilant capitalisme et marché, a clairement comme objectif opérationnel de perturber et d'entraver toute tentative de penser une alternative au capitalisme contemporain tel qu'il est, et à celui qu'ils rêvent pour demain, en tendant à cantonner ceux qui s'y consacrent, à la recherche, historiquement irréaliste, d'une alternative au marché.

Que tous ceux qui considèrent que le capitalisme est à ce jour le meilleur ou le moins mauvais des "systèmes" entretiennent l'assimilation du capitalisme et du marché peut, en abandonnant toute morale scientifique, apparaître logique. Mais serait-ce trop demander que ceux qui prétendent faire œuvre scientifique, que les économistes soucieux de ne pas ignorer l'histoire, et que ceux qui prétendent se consacrer à l'élaboration d'une éventuelle alternative au capitalisme contemporain, commencent par ne pas succomber à la confusion entretenue par la propagande capitaliste. Échapper à l'assimilation du capitalisme et du marché est, aujourd'hui, une condition nécessaire à toute pensée scientifiquement cohérente, et donc libre.

50 Le caractère fondamentalement centralisé du mode de coordination des décisions individuelles propre à l'univers walrassien a ainsi permis à l'économiste polonais Oskar Lange (1904-1965) d'élaborer un modèle cohérent de "socialisme de marché", en adossant la propriété publique des moyens de production à un système de marchés, et en confiant les fonctions du commissaire-priseur au... Bureau Central de Planification.

Voir : LANGE, O. On the Economic Theory of Socialism. Chicago. 1938.

51 Avatar : chacune des réincarnations successives de Vichnou. 


\section{BIBLIOGRAPHIE}

- BOURQUELOT, F. Études sur les foires de Champagne, sur la nature, l'étendue et les règles qui s'y faisaient aux XII ìme ${ }^{\text {XIII }}{ }^{\grave{m} e}$ et XIV $V^{\text {ème }}$ siècles. 2 volumes. Paris. Imprimerie Nationale. 1865-1866.

- BOYER, R. Une théorie du capitalisme est-elle possible? Paris. Odile Jacob. 2004.

- BRAUDEL, F. La Méditerranée et le monde méditerranéen à l'époque de Philippe II. 2 volumes. Paris. Armand Colin. 1966. (6 $6^{\mathrm{ème}}$ édition, 1986).

- BRAUDEL, F. Civilisation matérielle, économie et capitalisme. 3 volumes. Paris. Armand Colin. 1979.

- BRAUDEL, F. La dynamique du capitalisme. Paris. Arthaud. 1985.

- BRÉSARD, M. Les foires de Lyon aux XV ème et XVI ìme siècles. Paris. A. Picard. 1914.

- CAILLÉ, A. Dé-penser l'économique ; contre le fatalisme. Paris. La Découverte (MAUSS). 2005.

- CLOULAS, I. (coordination de). L'Italie de la Renaissance. Paris. Fayard. 1990.

- FAVIER, J. De l'or et des épices; naissance de l'homme d'affaires au Moyen Âge. Paris. Fayard. 1987.

- FITOUSSI, J-P. La démocratie et le marché. Paris. Grasset, Nouveau Collège de Philosophie. 2004.

- GODINHO, V. M. Les découvertes. $X V^{\text {ème }}-X V I^{\text {ème }}$ : une révolution des mentalités. Paris. Éditions Autrement, Série Mémoires, supplément au n¹. 1990.

- LANGE, O. On the Economic Theory of Socialism. Chicago. 1938

- MARGOLIN, J-C. L'avènement des Temps Modernes. Paris. P.U.F. 1977.

- MARX, K. Misère de la philosophie. Paris. Éditions Sociales. 1972.

- RENOUARD, Y. Les hommes d'affaires italiens du Moyen Âge. Paris. Armand Colin. 1949.

- SAPORI, A. Le marchand italien du Moyen Âge. Paris. Armand Colin. 1952.

- SARDELLA, P. Nouvelles et spéculations à Venise au début du XVI ème siècle. Paris. Armand Colin. 1948.

- SCHICK, L. Un grand homme d'affaires du XVİ̀me siècle : Jacob Fugger. Paris. S.E.V.P.E.N. 1957.

- VARILLE, M. Les foires de Lyon avant la Révolution. Lyon. L. Bonnaviat. 1920.

- VASQUEZ DE PRADA, V. Lettres marchandes d'Anvers. 4 volumes. Paris. S.E.V.P.E.N. 1960-1961.

- WALERY, S. Communication et accumulation du capital ; pour une perspective de longue durée. In : Quaderni. CREDAP. Université Paris-IX-Dauphine. Hiver 1990/1991. pp 15-35.

- WALERY, S. Les hommes d'affaires de la Renaissance et l' "esprit" du capitalisme: l'exemple de la lettre de change. Actes du Forum de la Régulation 2003. 